\title{
Informações Editoriais 2013
}

\section{Artigos Publicados - 2013}

\begin{tabular}{lcc}
\hline Edições & Autores por Edição & Artigos por Edição \\
\hline Jan/Mar, 2013 & 21 & 6 \\
\hline Abr/Jun, 2013 & 22 & 6 \\
\hline Jul/Set, 2013 & 17 & 6 \\
\hline Out/Dez, 2013 & 15 & 6 \\
\hline Total & 75 & 24 \\
\hline Média & 18,75 & 6 \\
\hline
\end{tabular}

Número de Artigos por Unidade de Federação - 2013

\begin{tabular}{|c|c|c|c|c|c|c|c|c|c|c|}
\hline \multirow{3}{*}{ Unidade da Federação } & \multicolumn{10}{|c|}{ Edições } \\
\hline & \multicolumn{2}{|c|}{ Jan/Mar } & \multicolumn{2}{|c|}{ Abr/Jun } & \multicolumn{2}{|c|}{ Jul/Set } & \multicolumn{2}{|c|}{ Out/Dez } & \multicolumn{2}{|c|}{ Total } \\
\hline & $\mathrm{Fi}$ & $\mathrm{Fi} \%$ & $\mathrm{Fi}$ & $\mathrm{Fi} \%$ & $\mathrm{Fi}$ & $\mathrm{Fi} \%$ & $\mathrm{Fi}$ & $\mathrm{Fi} \%$ & $\mathrm{Fi}$ & $\mathrm{Fi} \%$ \\
\hline Ceará & 1 & $17 \%$ & 1 & $17 \%$ & - & - & - & - & 2 & $8 \%$ \\
\hline Espírito Santo & - & - & 1 & $17 \%$ & 1 & $17 \%$ & 1 & $17 \%$ & 3 & $13 \%$ \\
\hline Minas Gerais & - & - & - & - & 1 & $17 \%$ & 1 & $17 \%$ & 2 & $8 \%$ \\
\hline Minas Gerais/ Paraná & 1 & $17 \%$ & - & - & - & - & - & - & 1 & $4 \%$ \\
\hline Paraíba & - & - & - & - & 1 & $17 \%$ & - & - & 1 & $4 \%$ \\
\hline Paraíba/ Rio Grando do Norte & - & - & - & - & - & - & 1,00 & $17 \%$ & 1 & $4 \%$ \\
\hline Paraná & 2 & $33 \%$ & 1 & $17 \%$ & - & - & - & - & 3 & $13 \%$ \\
\hline Piauí & - & - & - & - & - & - & 1 & $17 \%$ & 1 & $4 \%$ \\
\hline Rio Janeiro/Paraíba & - & - & - & - & 1 & $17 \%$ & - & - & 1 & $4 \%$ \\
\hline Rio Grande do Sul & 1 & $17 \%$ & - & - & - & - & - & - & 1 & $4 \%$ \\
\hline Santa Catarina & - & - & 1 & $17 \%$ & - & - & - & - & 1 & $4 \%$ \\
\hline $\begin{array}{l}\text { Santa Catarina/Mato Grosso/ } \\
\text { Paraná }\end{array}$ & - & - & - & - & 1 & $17 \%$ & - & - & 1 & $4 \%$ \\
\hline Santa Catarina/Paraná & 1 & $17 \%$ & 2 & $33 \%$ & - & - & 1 & $17 \%$ & 4 & $17 \%$ \\
\hline São Paulo & - & - & - & - & 1,00 & $17 \%$ & - & - & 1 & $4 \%$ \\
\hline São Paulo/Goiás/ Santa Catarina & - & - & - & - & - & - & 1 & $17 \%$ & 1 & $4 \%$ \\
\hline Total & 6 & $100 \%$ & 6 & $100 \%$ & 6 & $100 \%$ & 6 & $100 \%$ & 24 & $100 \%$ \\
\hline
\end{tabular}


Número de Autores por Unidade de Federação - 2013

\begin{tabular}{|c|c|c|c|c|c|c|c|c|c|c|}
\hline \multirow{3}{*}{ Unidade da Federação } & \multicolumn{10}{|c|}{ Edições } \\
\hline & \multicolumn{2}{|c|}{ Jan/Mar } & \multicolumn{2}{|c|}{ Abr/Jun } & \multicolumn{2}{|c|}{ Jul/Set } & \multicolumn{2}{|c|}{ Out/Dez } & \multicolumn{2}{|c|}{ Total } \\
\hline & $\mathrm{Fi}$ & Fi \% & $\mathrm{Fi}$ & $\mathrm{Fi} \%$ & $\mathrm{Fi}$ & $\mathrm{Fi} \%$ & $\mathrm{Fi}$ & $\mathrm{Fi} \%$ & $\mathrm{Fi}$ & $\mathrm{Fi} \%$ \\
\hline Ceará & 2 & $13 \%$ & 4 & $27 \%$ & - & - & - & - & 6 & $8 \%$ \\
\hline Espírito Santo & - & - & 3 & $20 \%$ & 2 & $13 \%$ & 2 & $13 \%$ & 7 & $9 \%$ \\
\hline Paraíba & - & - & - & - & 5 & $33 \%$ & 1 & $7 \%$ & 6 & $8 \%$ \\
\hline Mato Grosso & - & - & - & - & 1 & $7 \%$ & - & - & 1 & $1 \%$ \\
\hline Minas Gerais & 3 & $20 \%$ & - & - & 3 & $20 \%$ & 3 & $20 \%$ & 9 & $12 \%$ \\
\hline Paraná & 10 & $67 \%$ & 6 & $40 \%$ & - & - & 1 & $7 \%$ & 17 & $23 \%$ \\
\hline Piauí & - & - & - & - & - & - & 1 & $7 \%$ & 1 & $1 \%$ \\
\hline Goiás & - & - & - & - & - & - & 1 & $7 \%$ & 1 & $1 \%$ \\
\hline Rio de Janeiro & - & - & - & - & 2 & $13 \%$ & - & - & 2 & $3 \%$ \\
\hline Rio Grande do Sul & 4 & $27 \%$ & - & - & - & - & - & - & 4 & $5 \%$ \\
\hline Rio Grande do Norte & - & - & - & - & - & - & 1 & $7 \%$ & 1 & $1 \%$ \\
\hline Rondônia & - & - & - & - & 1 & $7 \%$ & - & - & 1 & $1 \%$ \\
\hline Santa Catarina & 2 & $13 \%$ & 9,00 & $60 \%$ & 2 & $13 \%$ & 4 & $27 \%$ & 17 & $23 \%$ \\
\hline São Paulo & - & - & - & - & 1 & $7 \%$ & 1 & $7 \%$ & 2 & $3 \%$ \\
\hline Total & 21 & $100 \%$ & 22 & $100 \%$ & 17 & $100 \%$ & 15 & $100 \%$ & 75 & $100 \%$ \\
\hline
\end{tabular}

Inventário dos Artigos - 2013

\begin{tabular}{lc}
\hline Itens & $\mathbf{2 0 1 3}$ \\
\hline Estoque inicial de artigos em $1^{\circ}$ de janeiro & 27 \\
$\quad$ Em avaliação & 17 \\
\hline$\quad$ Aprovados & 10 \\
\hline Número de artigos submetidos & 92 \\
\hline Número de artigos não aprovados no Desk Review & $(35)$ \\
\hline Número de artigos reprovados & $(35)$ \\
\hline Número de artigos publicados & $(24)$ \\
\hline Número de artigos aceitos para edições seguintes & $(1)$ \\
\hline Estoque Final de artigos em avaliação em 31 de dezembro & 24 \\
\hline
\end{tabular}


Instituições de Origem dos Autores dos Artigos - 2013

\begin{tabular}{|c|c|c|c|c|c|c|}
\hline \multirow{3}{*}{$\begin{array}{l}\text { UF/IES de origem } \\
\text { CE/UFC }\end{array}$} & \multicolumn{6}{|c|}{ Edições } \\
\hline & \multirow{2}{*}{$\frac{\text { Jan/Mar }}{2}$} & \multirow{2}{*}{$\frac{\text { Abr/Jun }}{4}$} & \multirow{2}{*}{$\frac{\text { Jul/Set }}{-}$} & \multirow{2}{*}{ Out/Dez } & \multicolumn{2}{|c|}{ Total } \\
\hline & & & & & 6 & $8 \%$ \\
\hline ES/FUCAPE & - & 3 & 2 & 2 & 7 & $9 \%$ \\
\hline GO/UFG & - & - & - & 1 & 1 & $1 \%$ \\
\hline MG/UFMG & 3 & - & 3 & 1 & 7 & $9 \%$ \\
\hline MG/FNH & - & - & - & 1 & 1 & $1 \%$ \\
\hline MT/UFMT & - & - & 1 & - & 1 & $1 \%$ \\
\hline PB/UFPB & - & - & 5 & 1 & 6 & $8 \%$ \\
\hline PR/PUC & 1 & - & - & - & 1 & $1 \%$ \\
\hline PR/UFPR & 3 & 3 & - & 1 & 7 & $9 \%$ \\
\hline PR/UTFPR & 4 & 1 & - & - & 5 & $7 \%$ \\
\hline PR/UEPR & 1 & - & - & - & 1 & $1 \%$ \\
\hline $\mathrm{PI} / \mathrm{FMN}$ & - & - & - & 1 & 1 & $1 \%$ \\
\hline RJ/UFRJ & - & - & 1 & - & 1 & $1 \%$ \\
\hline RN/UFERSA & - & - & - & 1 & 1 & $1 \%$ \\
\hline RO/UNEC & - & - & 1 & - & 1 & $1 \%$ \\
\hline RS/UNISINOS & 4 & - & - & - & 4 & $5 \%$ \\
\hline SC/UFSC & 2 & 2 & - & - & 4 & $5 \%$ \\
\hline SC/FURB & - & 5 & 1 & 1 & 7 & $9 \%$ \\
\hline SC/UDESC & - & 2 & - & - & 2 & $3 \%$ \\
\hline SP/USP & - & - & 1 & 1 & 2 & $3 \%$ \\
\hline Outras Instituições & 1 & 2 & 2 & 4 & 9 & $12 \%$ \\
\hline Total & 21 & 22 & 17 & 15 & 75 & $100 \%$ \\
\hline
\end{tabular}




\section{Periodo Médio de Circulação dos Artigos Publicados - 2013}

Vol. 7, n. 1, 2013

\begin{tabular}{|c|c|c|c|c|c|c|c|c|c|}
\hline $\begin{array}{l}N^{\circ} \text {. do } \\
\text { Artigo }\end{array}$ & $\begin{array}{c}\text { Data de } \\
\text { submissão }\end{array}$ & $\begin{array}{l}\text { Avaliado } \\
\text { pelos } \\
\text { Pares }\end{array}$ & $\begin{array}{l}\text { Reformulado } \\
\text { pelos autores }\end{array}$ & $\begin{array}{c}\text { Data da } \\
\text { Aceitação }\end{array}$ & $\begin{array}{c}\text { Data da } \\
\text { Publicação }\end{array}$ & $\begin{array}{c}\mathrm{N}^{\circ} \text {. de } \\
\text { dias para } \\
\text { avaliação }\end{array}$ & $\begin{array}{c}\mathrm{N}^{\circ} \text {. de } \\
\text { dias para } \\
\text { reformulação }\end{array}$ & $\begin{array}{c}\mathbf{N}^{\circ} \text {. de } \\
\text { dias para } \\
\text { recomendação } \\
\text { de publicação }\end{array}$ & $\begin{array}{c}\mathbf{N}^{\circ} \text {. de dias } \\
\text { entre a } \\
\text { submissão e } \\
\text { publicação }\end{array}$ \\
\hline 1 & 01/07/2011 & $11 / 02 / 2012$ & $14 / 02 / 2012$ & $10 / 06 / 2012$ & 29/03/2013 & 220 & 3 & 116 & 628 \\
\hline 2 & $04 / 12 / 2010$ & $13 / 07 / 2011$ & $30 / 08 / 2011$ & $21 / 09 / 2011$ & 29/03/2013 & 219 & 47 & 21 & 835 \\
\hline 3 & $20 / 12 / 2010$ & $12 / 12 / 2011$ & $18 / 01 / 2012$ & $18 / 05 / 2012$ & 29/03/2013 & 352 & 36 & 120 & 819 \\
\hline 4 & $02 / 03 / 2010$ & $18 / 10 / 2010$ & 15/09/2011 & 23/09/2011 & 29/03/2013 & 226 & 327 & 8 & 1107 \\
\hline 5 & $13 / 04 / 2010$ & $18 / 04 / 2011$ & $27 / 05 / 2011$ & $12 / 12 / 2011$ & $29 / 03 / 2013$ & 365 & 39 & 195 & 1066 \\
\hline 6 & 05/01/2011 & $01 / 02 / 2012$ & 08/02/2012 & $21 / 02 / 2012$ & $29 / 03 / 2013$ & 386 & 7 & 13 & 804 \\
\hline \multicolumn{5}{|c|}{ Média de dias para publicação de artigos por Edição } & & 309,6 & & & 876,5 \\
\hline \multicolumn{6}{|c|}{ Média de meses para publicação de artigos por Edição } & 10,3 & & & 29,2 \\
\hline
\end{tabular}

Vol. 7, n. 2, 2013

\begin{tabular}{|c|c|c|c|c|c|c|c|c|c|}
\hline $\begin{array}{l}\mathrm{N}^{\circ} \text {. do } \\
\text { Artigo }\end{array}$ & $\begin{array}{c}\text { Data de } \\
\text { submissão }\end{array}$ & $\begin{array}{c}\text { Avaliado } \\
\text { pelos } \\
\text { Pares }\end{array}$ & $\begin{array}{l}\text { Reformulado } \\
\text { pelos autores }\end{array}$ & $\begin{array}{c}\text { Data da } \\
\text { Aceitação }\end{array}$ & $\begin{array}{c}\text { Data da } \\
\text { Publicação }\end{array}$ & $\begin{array}{c}\mathbf{N}^{\circ} \text {. de } \\
\text { dias para } \\
\text { avaliação }\end{array}$ & $\begin{array}{c}\mathrm{N}^{\circ} \text {. de } \\
\text { dias para } \\
\text { reformulação }\end{array}$ & $\begin{array}{c}\mathbf{N}^{\circ} \text {. de } \\
\text { dias para } \\
\text { recomendação } \\
\text { de publicação }\end{array}$ & $\begin{array}{c}\mathrm{N}^{\circ} \text {. de dias } \\
\text { entre a } \\
\text { submissão e } \\
\text { publicação }\end{array}$ \\
\hline 1 & 02/05/2010 & $02 / 01 / 2012$ & 13/05/2012 & $23 / 09 / 2012$ & $28 / 06 / 2013$ & 600 & 131 & 130 & 1136 \\
\hline 2 & $17 / 11 / 2011$ & $02 / 02 / 2012$ & $13 / 03 / 2012$ & $11 / 07 / 2012$ & $28 / 06 / 2013$ & 75 & 41 & 118 & 581 \\
\hline 3 & $21 / 12 / 2011$ & 06/03/2012 & 20/09/2012 & $17 / 10 / 2012$ & $28 / 06 / 2013$ & 75 & 194 & 27 & 547 \\
\hline 4 & $21 / 07 / 2011$ & 29/10/2011 & 08/11/2011 & $11 / 11 / 2011$ & $28 / 06 / 2013$ & 98 & 9 & 3 & 697 \\
\hline 5 & $30 / 04 / 2013$ & $14 / 05 / 2013$ & $26 / 05 / 2013$ & $27 / 05 / 2013$ & $28 / 06 / 2013$ & 14 & 12 & 1 & 58 \\
\hline 6 & $12 / 01 / 2012$ & 08/10/2012 & 29/10/2012 & $01 / 01 / 2013$ & $28 / 06 / 2013$ & 266 & 21 & 62 & 526 \\
\hline \multicolumn{6}{|c|}{ Média de dias para publicação de artigos por Edição } & 105,6 & & & 590,8 \\
\hline \multicolumn{6}{|c|}{ Média de meses para publicação de artigos por Edição } & 3,5 & & & 19,7 \\
\hline
\end{tabular}


Vol. 7, n. 3, 2013

\begin{tabular}{|c|c|c|c|c|c|c|c|c|c|}
\hline $\begin{array}{l}\mathbf{N}^{\circ} \text {. do } \\
\text { Artigo }\end{array}$ & $\begin{array}{c}\text { Data de } \\
\text { submissão }\end{array}$ & $\begin{array}{c}\text { Avaliado } \\
\text { pelos } \\
\text { Pares }\end{array}$ & $\begin{array}{l}\text { Reformulado } \\
\text { pelos autores }\end{array}$ & $\begin{array}{c}\text { Data da } \\
\text { Aceitação }\end{array}$ & $\begin{array}{c}\text { Data da } \\
\text { Publicação }\end{array}$ & $\begin{array}{c}\text { No. de } \\
\text { dias para } \\
\text { avaliação }\end{array}$ & $\begin{array}{c}\mathrm{N}^{\circ} \text {. de } \\
\text { dias para } \\
\text { reformulação }\end{array}$ & $\begin{array}{c}\mathbf{N}^{\circ} \text {. de } \\
\text { dias para } \\
\text { recomendação } \\
\text { de publicação }\end{array}$ & $\begin{array}{c}\mathbf{N}^{\circ} \text {. de dias } \\
\text { entre a } \\
\text { submissão e } \\
\text { publicação }\end{array}$ \\
\hline 1 & 06/11/2012 & 04/03/2013 & 19/06/2013 & $25 / 07 / 2013$ & $30 / 09 / 2013$ & 118 & 105 & 36 & 324 \\
\hline 2 & 22/02/2013 & 29/04/2013 & 06/05/2013 & 09/05/2013 & $30 / 09 / 2013$ & 67 & 7 & 3 & 218 \\
\hline 3 & 08/07/2013 & 18/08/2013 & $25 / 08 / 2013$ & $27 / 08 / 2013$ & $30 / 09 / 2013$ & 40 & 7 & 2 & 82 \\
\hline 4 & $22 / 07 / 2013$ & 20/08/2013 & $25 / 08 / 2013$ & $30 / 08 / 2013$ & $30 / 09 / 2013$ & 28 & 5 & 5 & 68 \\
\hline 5 & 03/04/2013 & $21 / 05 / 2013$ & $01 / 08 / 2013$ & 08/08/2013 & $30 / 09 / 2013$ & 48 & 70 & 7 & 177 \\
\hline 6 & $01 / 07 / 2013$ & 24/08/2013 & 05/09/2013 & 08/09/2013 & $30 / 09 / 2013$ & 53 & 11 & 3 & 89 \\
\hline \multicolumn{5}{|c|}{ Média de dias para publicação de artigos por Edição } & & 47,2 & & & 159,7 \\
\hline \multicolumn{6}{|c|}{ Média de meses para publicação de artigos por Edição } & 1,6 & & & 5,3 \\
\hline
\end{tabular}

Vol. 7, n. 4, 2013

\begin{tabular}{|c|c|c|c|c|c|c|c|c|c|}
\hline $\begin{array}{l}\mathbf{N}^{\circ} \text {. do } \\
\text { Artigo }\end{array}$ & $\begin{array}{c}\text { Data de } \\
\text { submissão }\end{array}$ & $\begin{array}{c}\text { Avaliado } \\
\text { pelos } \\
\text { Pares }\end{array}$ & $\begin{array}{l}\text { Reformulado } \\
\text { pelos autores }\end{array}$ & $\begin{array}{c}\text { Data da } \\
\text { Aceitação }\end{array}$ & $\begin{array}{c}\text { Data da } \\
\text { Publicação }\end{array}$ & $\begin{array}{c}N^{\circ} \text {. de } \\
\text { dias para } \\
\text { avaliação }\end{array}$ & $\begin{array}{c}\mathbf{N}^{\circ} \text {. de } \\
\text { dias para } \\
\text { reformulação }\end{array}$ & $\begin{array}{c}\mathbf{N}^{\circ} \text {. de } \\
\text { dias para } \\
\text { recomendação } \\
\text { de publicação }\end{array}$ & $\begin{array}{c}\mathbf{N}^{\circ} \text {. de dias } \\
\text { entre a } \\
\text { submissão e } \\
\text { publicação }\end{array}$ \\
\hline 1 & $12 / 06 / 2013$ & 26/07/2013 & $21 / 08 / 2013$ & 08/09/2013 & 20/12/2013 & 44 & 25 & 17 & 188 \\
\hline 2 & 07/08/2013 & $21 / 09 / 2013$ & 02/10/2013 & 05/10/2013 & 20/12/2013 & 44 & 11 & 3 & 133 \\
\hline 3 & 04/07/2013 & $24 / 08 / 2013$ & $17 / 09 / 2013$ & $21 / 09 / 2013$ & 20/12/2013 & 50 & 23 & 4 & 166 \\
\hline 4 & $29 / 06 / 2013$ & 17/08/2013 & $01 / 09 / 2013$ & 08/09/2013 & 20/12/2013 & 48 & 14 & 7 & 171 \\
\hline 5 & $14 / 07 / 2013$ & $19 / 08 / 2013$ & 03/09/2013 & 08/09/2013 & $20 / 12 / 2013$ & 35 & 14 & 5 & 156 \\
\hline 6 & $16 / 06 / 2013$ & $22 / 08 / 2013$ & $13 / 08 / 2013$ & $23 / 10 / 2013$ & $20 / 12 / 2013$ & 66 & -9 & 70 & 184 \\
\hline \multicolumn{5}{|c|}{ Média de dias para publicação de artigos por Edição } & & 48,6 & & & 166,3 \\
\hline \multicolumn{5}{|c|}{ Média de meses para publicação de artigos por Edição } & & 1,6 & & & 5,5 \\
\hline
\end{tabular}

\begin{tabular}{lcc}
\hline Média de dias para publicação de artigos por Edição em 2013 & 102,0 & 339,7 \\
\hline Média de meses para publicação de artigos por Edição em 2013 & 3,4 \\
\hline
\end{tabular}




\section{Avaliadores Ad Hoc e Instituição de Vínculo - 2013}

\begin{tabular}{|c|c|}
\hline Avaliador & Instituição \\
\hline Adriano Rodrigues & Universidade Federal do Rio de Janeiro \\
\hline Alessandra Vasconcelos Gallon & Universidade Federal do Ceará \\
\hline Alexandre Rivas Rivas & Universidade Federal do Amazonas \\
\hline Alfredo Sarlo Neto & Universidade Federal do Espírito Santo \\
\hline Alvaro Augusto Ricardino Filho & Pontifícia Universidade Católica de São Paulo \\
\hline Amaury José Rezende & Universidade de São Paulo - Ribeirão Preto \\
\hline Ana Carolina Pimentel Duarte da Fonseca & Universidade Federal do Rio de Janeiro \\
\hline Ana Cristina de Faria & Universidade Municipal de São Caetano do Sul \\
\hline Ana Gisbert (Espanha) & Universidade Autonoma de Madrid - Espanha \\
\hline Ana Lúcia Fontes Souza Vasconcelos & Universidade Federal de Pernambuco \\
\hline Antonia Silva de Lima & Universidade Federal do Amazonas \\
\hline Antonio Artur de Souza & Universidade Federal de Minas Gerais \\
\hline Antonio Carlos Dias Coelho & Universidade Federal do Ceará \\
\hline Antonio Gonçalves de Oliveira & Universidade Tecnológica Federal do Paraná \\
\hline Antonio Lopo Martinez & Fucape Business School \\
\hline Aridelmo José Campanharo Teixeira & Fucape Business School \\
\hline Arilda Magda Campagnaro Teixeira & Fucape Business School \\
\hline Artur Roberto do Nascimento & Universidade Estadual de Feira de Santana \\
\hline Bruno Felix Von Borell de Araujo & Fucape Business School \\
\hline Bruno Funchal & Fucape Business School \\
\hline Cacilda Andrade & Universidade Federal de Pernambuco \\
\hline Carlos Renato Theóphilo & Universidade Estadual de Montes Claros \\
\hline Carlos Roberto Godoy & Universidade de São Paulo - Ribeirão Preto \\
\hline César Augusto Tiburcio Silva & Universidade de Brasília \\
\hline Claudio de Souza Miranda & Universidade de São Paulo - Ribeirão Preto \\
\hline Claudio Parise & Fundação Escola de Comércio Álvares Penteado \\
\hline Daphins Teodoro da Silva Junior & Universidade de São Paulo \\
\hline Deisy Cristina Corrêa Igarashi & Universidade Estadual do Oeste do Paraná \\
\hline Dione Olesczuk Soutes & Universidade Estadual do Oeste do Paraná \\
\hline Edgard B. Cornachione Jr & Universidade de São Paulo \\
\hline Edilene Santos Santana & Fundação Getúlio Vargas - São Paulo \\
\hline Edílson Paulo & Universidade Federal da Paraíba \\
\hline Eliseu Martins & Universidade de São Paulo \\
\hline Ernani Ott & Universidade do Vale do Rio dos Sinos \\
\hline Fábio Frezatti & Universidade de São Paulo \\
\hline Fabio Gallo Garcia & Fundação Getúlio Vargas - São Paulo \\
\hline
\end{tabular}




\begin{tabular}{|c|c|}
\hline Fábio Moraes da Costa & Fucape Business School \\
\hline Felipe Ramos Ferreira & Fucape Business School \\
\hline Fernanda Filgueiras Sauerbronn & Universidade Federal do Rio de Janeiro \\
\hline Fernando Caio Galdi & Fucape Business School \\
\hline Fernando Dal-Ri Murcia & Universidade Federal de Santa Catarina \\
\hline Fernando de Almeida Santos & Pontifícia Universidade Católica de São Paulo \\
\hline Flávia Zóboli Dalmácio & Universidade de São Paulo \\
\hline Francisco Antonio Bezerra & Fundação Universidade Regional de Blumenau \\
\hline Francisco Carlos Fernandes & Fundação Universidade Regional de Blumenau \\
\hline Gerlando Augusto Sampaio Franco de Lima & Universidade de São Paulo \\
\hline Guillermo Braunbeck & Fundação Instituto de Pesquisas Contábeis, Atuariais e Financeiras \\
\hline Henrique Formigoni & Universidade Presbiteriana Mackenzie \\
\hline Ilse Maria Beuren & Universidade Federal do Paraná \\
\hline Ivam Carlos Peleias & Fundação Escola de Comércio Álvares Penteado \\
\hline Jacqueline Veneroso Alves da Cunha & Universidade Federal de Minas Gerais \\
\hline Jaime Crozatti & Universidade de São Paulo \\
\hline Jeronymo José Libonati & Universidade Federal de Pernambuco \\
\hline Joanilia Neide de Sales Cia. & Universidade de São Paulo \\
\hline Jorge de Souza Bispo & Receita Federal do Brasil \\
\hline Jorge Eduardo Scarpin & Universidade Federal do Paraná \\
\hline Jorge Vieira Costa Junior & Universidade do Estado do Rio de Janeiro \\
\hline Jose Alexandre Magrini Pigatto & Universidade Federal de Santa Maria \\
\hline José Alonso Borba & Universidade Federal de Santa Catarina \\
\hline José Ellias Feres de Almeida & Universidade Federal do Espírito santo \\
\hline José Maria Dias Filho & Universidade Federal da Bahia \\
\hline José Roberto Kassai & Universidade de São Paulo \\
\hline JOSHUA ONOME IMONIANA & Universidade Presbiteriana Mackenzie \\
\hline Joshua Onome Inoniama & Universidade Presbiteriana Mackenzie \\
\hline Josilmar Codernonssi Cia, & Universidade Presbiteriana Mackenzie \\
\hline Laura Calixto & Universidade Federal de São Paulo \\
\hline Laura Edith Taboada Pinheiro & Universidade Federal de Minas Gerais \\
\hline Luciano Gomes Reis & Universidade Estadual de Londrina \\
\hline Luiz Alberton & Universidade Federal de Santa Catarina \\
\hline Luiz Eduardo Afonso & Universidade de São Paulo \\
\hline Luiza Maria Bessa Rebelo & Universidade Federal do Amazonas \\
\hline Maisa de Souza Ribeiro & Universidade de São Paulo - Ribeirão Preto \\
\hline Marcelle Colares Oliveira & Universidade Federal do Ceará \\
\hline
\end{tabular}




\begin{tabular}{|c|c|}
\hline Marcelo Alvaro da Silva Macedo & Universidade Federal do Rio de Janeiro \\
\hline Marcia Maria dos Santos Bortolocci Espejo & Universidade Federal do Paraná \\
\hline Marcos Antonio de Souza & Universidade do Vale do Rio dos Sinos \\
\hline Marcus Vinicius Veras Machado & Universidade Federal do Ceará \\
\hline Maria José Carvalho de Souza Domingues & Fundação Universidade Regional de Blumenau \\
\hline Mariana Simões Ferrazdo Amaral Fregonesi & Universidade de São Paulo \\
\hline Mayla Cristina Costa & Universidade Federal do Paraná \\
\hline Monica Sionara Schpallir Calijuri & Universidade Presbiteriana Mackenzie \\
\hline Neusa Maria Bastos Fernandes dos Santos & Pontifícia Universidade Católica de São Paulo \\
\hline Osmar Siena Siena & Universidade Federal de Rondônia \\
\hline Patricia Gonzalez Gonzalez & Universidade del Valle (Colômbia) \\
\hline Patrícia Siqueira Varela & Fundação Universidade Regional de Blumenau \\
\hline Paulo Roberto Barbosa Lustosa & Universidade de Brasília \\
\hline Raimundo Nonato Rodrigues & Universidade Federal de Pernambuco \\
\hline Renê Coppe Pimentel & Fucape Business School \\
\hline Renê Coppe Pimentel & Fucape Business School \\
\hline Rogério João Lunkes & Universidade Federal de Santa Catarina \\
\hline Romualdo Douglas Colauto & Universidade Federal do Paraná \\
\hline Silvio Hiroshi Nakao & Universidade de São Paulo - Ribeirão Preto \\
\hline Solange Garcia dos Reis & Universidade de São Paulo - Ribeirão Preto \\
\hline Sonia Maria da Silva Gomes & Universidade Federal da Bahia \\
\hline Sylvio Mario Pulga Ferreira & Universidade Federal do Amazonas \\
\hline Umbelina Cravo Texeira Lagioia & Universidade Federal de Pernambuco \\
\hline Valcemiro Nossa & Fucape Business School \\
\hline Vera Maria Rodrigues Ponte & Universidade Federal do Ceará \\
\hline Victor Branco de Holanda & Universidade Federal do Rio Grande do Norte \\
\hline Vilma Geni Slomski & Fundação Escola de Comércio Alvares Penteado \\
\hline Vinicius Aversari Martins & Universidade de São Paulo - Ribeirão Preto \\
\hline Zaina Said El Hajj & Universidade Federal do Rio de Janeiro \\
\hline
\end{tabular}


Origem dos Avaliadores - 2013

\begin{tabular}{lcc}
\hline Estado & Quantidade & Percentual \\
\hline Amazonas & 4 & $3,9 \%$ \\
\hline Bahia & 3 & $2,9 \%$ \\
\hline Ceará & 5 & $4,9 \%$ \\
\hline Distrito Federal & 2 & $2,0 \%$ \\
\hline Espirito Santo & 13 & $12,7 \%$ \\
\hline Minas Gerais & 4 & $3,9 \%$ \\
\hline Paraiba & 1 & $1,0 \%$ \\
\hline Pernambuco & 5 & $4,9 \%$ \\
\hline Paraná & 9 & $8,8 \%$ \\
\hline Rio de Janeiro & 6 & $5,9 \%$ \\
\hline Rio Grande do Norte & 1 & $1,0 \%$ \\
\hline Rondônia & 1 & $1,0 \%$ \\
\hline Rio Grande do Sul & 3 & $2,9 \%$ \\
\hline Santa Catarina & 8 & $7,8 \%$ \\
\hline São Paulo & 35 & $34,3 \%$ \\
\hline Total & 102 & $100 \%$ \\
\hline
\end{tabular}


Instituições de Lotação dos Avaliadores - 2013

\begin{tabular}{|c|c|c|c|}
\hline Instituição & Estado & Quantidade & Percentual \\
\hline Universidade Federal do Amazonas & AM & 4 & $3,9 \%$ \\
\hline Universidade Estadual de Feira de Santana & BA & 1 & $1,0 \%$ \\
\hline Universidade Federal da Bahia & BA & 2 & $2,0 \%$ \\
\hline Universidade Federal do Ceará & CE & 5 & $4,9 \%$ \\
\hline Universidade de Brasília & DF & 2 & $2,0 \%$ \\
\hline Fucape Business School & ES & 11 & $10,8 \%$ \\
\hline Universidade Federal do Espírito Santo & ES & 2 & $2,0 \%$ \\
\hline Universidade Estadual de Montes Claros & MG & 1 & $1,0 \%$ \\
\hline Universidade Federal de Minas Gerais & MG & 3 & $2,9 \%$ \\
\hline Universidade Federal da Paraíba & PB & 1 & $1,0 \%$ \\
\hline Universidade Federal de Pernambuco & PE & 5 & $4,9 \%$ \\
\hline Universidade Estadual de Londrina & PR & 1 & $1,0 \%$ \\
\hline Universidade Estadual do Oeste do Paraná & PR & 2 & $2,0 \%$ \\
\hline Universidade Federal do Paraná & PR & 5 & $4,9 \%$ \\
\hline Universidade Tecnológica Federal do Paraná & PR & 1 & $1,0 \%$ \\
\hline Universidade Estadual do Rio de Janeiro & RJ & 1 & $1,0 \%$ \\
\hline Universidade Federal do Rio de Janeiro & RJ & 5 & $4,9 \%$ \\
\hline Universidade Federal do Rio Grande do Norte & RN & 1 & $1,0 \%$ \\
\hline Universidade Federal de Rondônia & $\mathrm{RO}$ & 1 & $1,0 \%$ \\
\hline Universidade do Vale do Rio dos Sinos & RS & 2 & $2,0 \%$ \\
\hline Universidade Federal de Santa Maria & RS & 1 & $1,0 \%$ \\
\hline Fundação Universidade Regional de Blumenau & SC & 4 & $3,9 \%$ \\
\hline Universidade Federal de Santa Catarina & SC & 4 & $3,9 \%$ \\
\hline Fipecafi & SP & 1 & $1,0 \%$ \\
\hline Fundação Escola de Comércio Álvares Penteado & SP & 3 & $2,9 \%$ \\
\hline Fundação Getúlio Vargas - SP & SP & 2 & $2,0 \%$ \\
\hline Pontifícia Universidade Católica de São Paulo & SP & 3 & $2,9 \%$ \\
\hline Receita Federal do Brasil & SP & 1 & $1,0 \%$ \\
\hline Universidade de São Paulo & SP & 11 & $10,8 \%$ \\
\hline Universidade de São Paulo de Ribeirão Preto & SP & 7 & $6,9 \%$ \\
\hline Universidade Federal de São Paulo & SP & 1 & $1,0 \%$ \\
\hline Universidade Municipal de São Caetano do Sul & SP & 1 & $1,0 \%$ \\
\hline Universidade Presbiteriana Mackenzie & SP & 5 & $4,9 \%$ \\
\hline Universidade del Valle (Colômbia) & Colômbia & 1 & $1,0 \%$ \\
\hline Universidade Autonoma de Madrid & Espanha & 1 & $1,0 \%$ \\
\hline Total & & 102 & $100 \%$ \\
\hline
\end{tabular}

